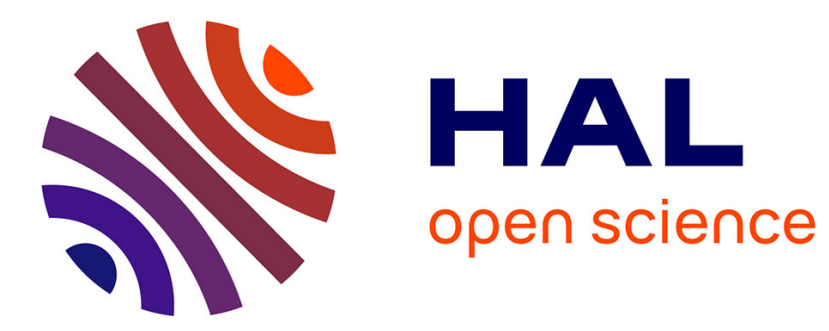

\title{
PROPRIETES NON LINEAIRES D'UN VERRE FLINT DENSE
}

\author{
Jean-Michel Rouvaen, G. Waxin, R. Torguet, E. Bridoux
}

\section{To cite this version:}

Jean-Michel Rouvaen, G. Waxin, R. Torguet, E. Bridoux. PROPRIETES NON LINEAIRES D'UN VERRE FLINT DENSE. Journal de Physique Colloques, 1979, 40 (C8), pp.C8-179-C8-182. 10.1051/jphyscol:1979830 . jpa-00219535

\section{HAL Id: jpa-00219535 https://hal.science/jpa-00219535}

Submitted on 1 Jan 1979

HAL is a multi-disciplinary open access archive for the deposit and dissemination of scientific research documents, whether they are published or not. The documents may come from teaching and research institutions in France or abroad, or from public or private research centers.
L'archive ouverte pluridisciplinaire HAL, est destinée au dépôt et à la diffusion de documents scientifiques de niveau recherche, publiés ou non, émanant des établissements d'enseignement et de recherche français ou étrangers, des laboratoires publics ou privés. 


\title{
PROPRIETES NON LINEAIRES ELASTIQUES D'UN VERRE FLINT DENSE
}

\author{
J.M. Rouvaen, G. Waxin, R. Torguet, E. Bridoux \\ Laboratoire d'Optoacoustoelectronique ERA $n^{\circ} 593$ C.N.R.S. Université de Valenciennes - 59326 \\ VaZenciennes Cédex - France.
}

\begin{abstract}
Rēsumẽ, - Le verre flint dense SF-59 de la Compagnei Schott est connu pour être un milieu fortement photoélastique. L'atténuation élevée de ce matériau permet de l'utiliser uniquement pour réaliser des modulateurs acousto-optiques.

L'atténuation élevêe des ondes longitudinales provient pour partie d'un excès d'atténuation dépendant de la puissance d'entrée, d'origine non linéaire. La propagation non linéaire d'ondes êlastiques longitudinales dans le verre SF-59 a été étudiẻe expérimentalement et les résultats principaux sont reportés.

Abstract. - The dense flint SF-59 Schott's glass is known as a highly acousto-optic materail. Owing to its high measured attenuation, it is only suited for acousto-optic modulator applications. The previously mentionned high longitudinal wave attenuation comes in part from the non-crystalline nature of the material and in part from an input power dependant non-linear excess attenuation. The nonlinear propagation of longitudinal elastic waves in the SF-59 glass has been studied experimentally and the main results are reported.
\end{abstract}

1. INTRODUCTION. - Les verres "flint" très denses possēdent gēnéralement des propriētés photoélastiques intéressantes / $1 /$ et sont utilisés dans nombre d'applications en acousto-optique $/ 2 /$. La vitesse de propagation des ondes acoustiques longitudinales et leur attenuation ont été mesurées pour un certain nombre de ces verres $/ 3 /$. Le représentant le plus intêressant de cette famille de verres est celui commercialisé sous la référence SF-59 par la compagnie Schott Glasswerk. Il possède en effet un facteur de mérite-acousto-optique /4/ êlevê, mais son attēnuation est très forte, ce qui limite les applications au domaine des modulateurs de lumière.

Dans un travail antérieur, /5/ nous avons suggeré l'existence d'une certaine corrêlation entre les propriētés acousto-optique et les propriétés non linéaires êlastiques, représentées par un facteur de mërite non linéaire. Les mèmes matériaux devraient donc convenir pour les deux types d'applications, aussi nous sommes nous intéressês aux propriétês non linēaires élastiques des verres "flint" très denses et, plus particulièrement, à celles du verre SF-59 que nous reportons ici.

\section{PROPAGATION NON LINEAIRE DES ONDES LONGITUDINA-} LES DANS LE VERRE SF-59. - La détection de 1'amp1itude et de 1 a phase des faisceaux acoustiques au moyen d'une sonde laser constitue une méthode d'étude idéale de leur propagation, car elle nécessite pas de contact mécanique et $n$ 'introduit aucune perturbation $/ 6 /$.

Nous avons, à l'aide d'un tel instrument, mesuré la vitesse des ondes acoustiques longitudinales dans le verre SF-59

A cet effet, nous avons enregistré la phase d'une onde acoustique longitudinale en fonction de la distance de propagation. Un capteur de déplacement de linéarité excellent, voisine de $1 \%$, est utilisé pour mesurer le déplacement 1 de l'équipage mécanique supportant la ligne acoustique devant le faisceau laser focalisé (voir figure 1): Si ce déplacement correspond exactement $\bar{a}$ un nombre entier $n$ de fois la longueur d'onde acoustique $\Lambda$ à la fréquence de travail $f$, mesurée avec six chiffres sigificatifs à l'aide d'un fréquencemètre, la vitesse de 1 'onde acoustique est donné par

$$
v=\Lambda f=1 \mathrm{f} / \mathrm{n}
$$

On peut attendre de cette méthode de mesure une précision globale de 1 'ordre de $1 \%$. Nous avons trouvé une vàleur

$$
v_{L}=3170 \pm 2 m / s
$$

assez proche de celle donnée dans la littērature / $3 /$ $13 /$, dêduite de la mesure plus grossière de l'angle de BRAGG

$$
\Phi_{\theta}=\lambda / 2 \Lambda
$$

où est la longueur d'onde lumineuse dans le vide 


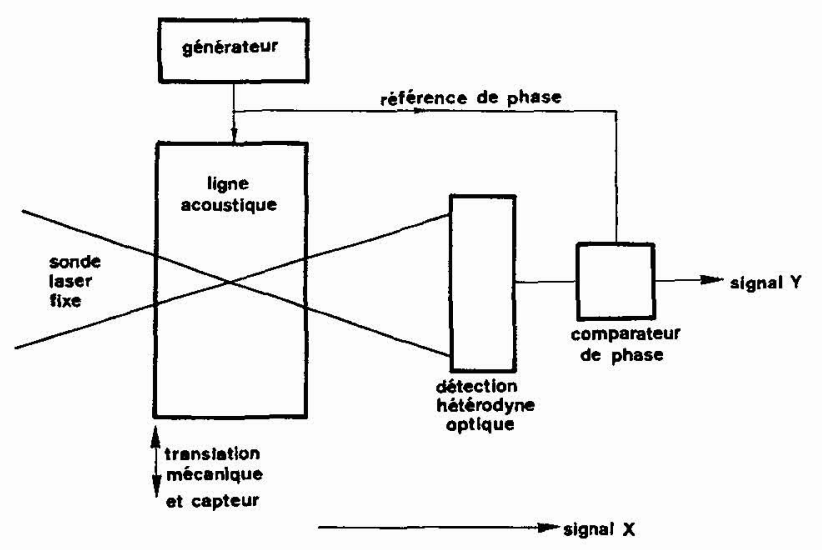

Fig. 1 Mesure de vitesse a - Principe

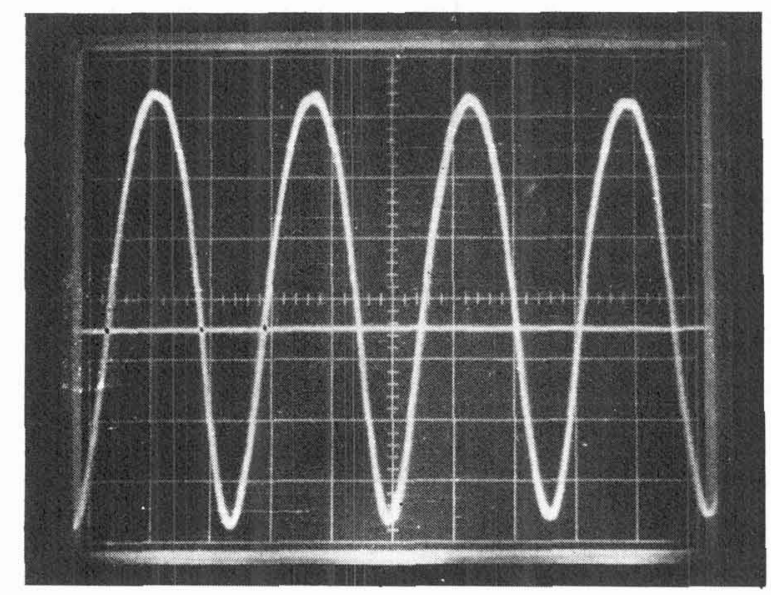

b - Enregistrement de la phase de l'onde acoustique

à partir de la distance séparant deux faisceaux lumineux dans un plan parallèle au faisceau acoustique (voir figure 2).

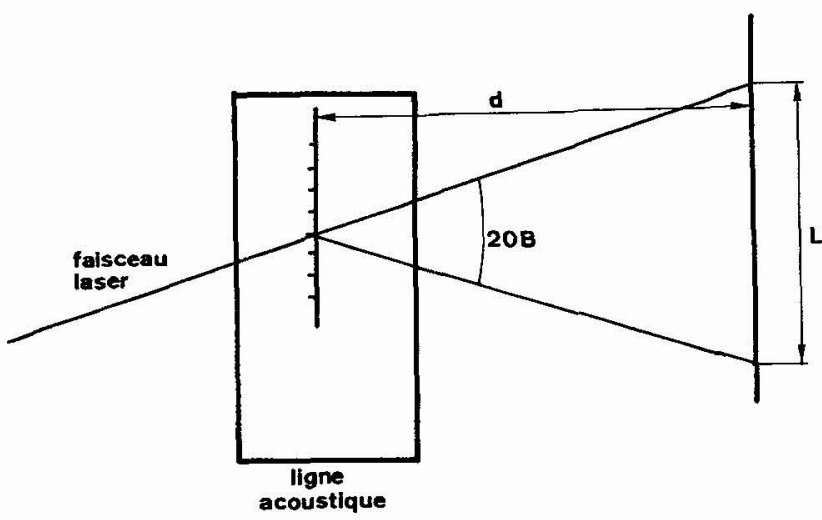

Fig. 2 : Mesure de 1'angle de BRAGG

La longueur d'onde acoustique est déduite de la mesure de $L=2 d \operatorname{tg} B$
Nous avons ensuite étudiē l'attēnuation des ondes acoustiques longitudinales en relevant les variations de 1 'amplitude en fonction de la distance parcourue dans la ligne acoustique. Nous avons ainsi constaté que l'attênuation est fonction de la puissance acoustique, ce qui est l'indice d'effets non linéaires se produisant au cours de la propagation.

Les résultats obtenus, pour une fréquence opératoire égale à $26 \mathrm{MHz}$, sont donnés à la figure 3 , pour les ondes acoustiques fondamentale et harmonique de rang 2.

L'atténuation élevêe des ondęs longitudinales provient non seulement de la nature polycristalline du matériau, mais aussi de l'excès d'atténuation dû aux effets non linéaires, et ceci pour une part non négligeable. La figure 4 reprêsente l'êvolution de la puissance des ondes acoustiques harmoniques en fonction de la distance de propagation.

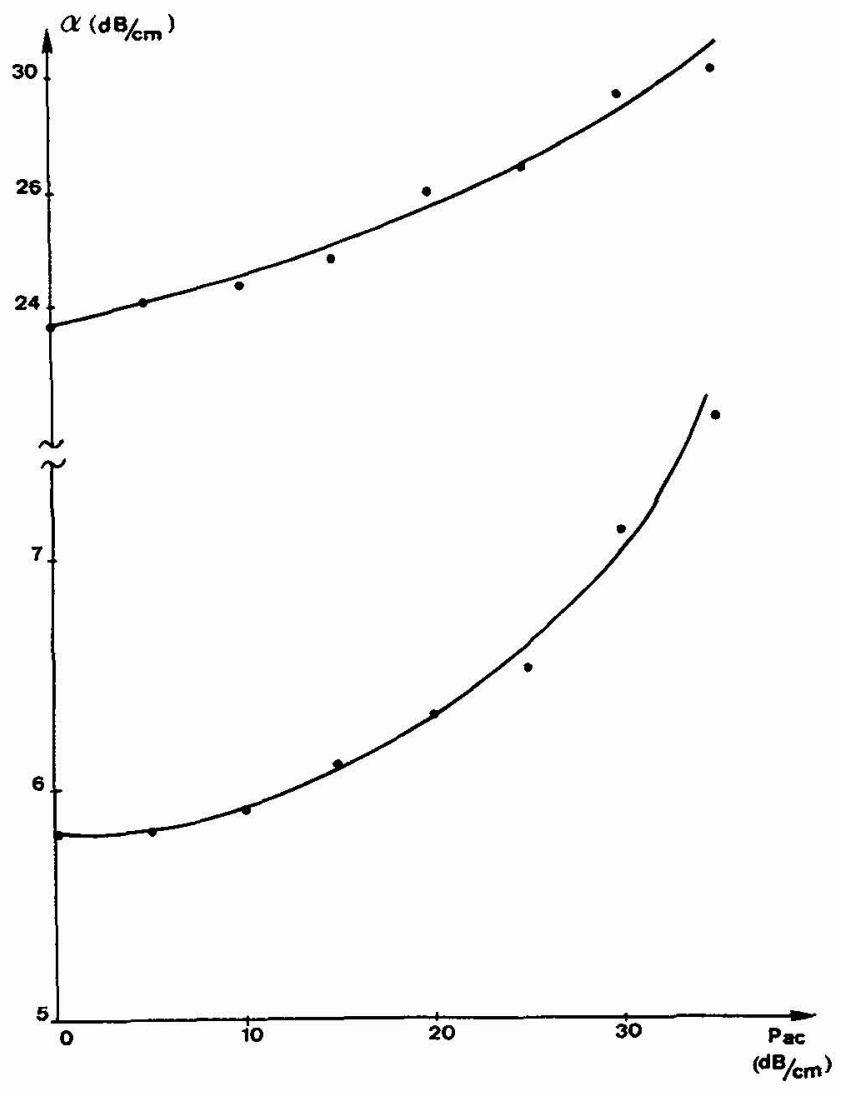

Fig. 3 Atténuation des ondes longitudinales en fonction de la puissance acoustique au niveau du transducteur

I1 convient de remarquer que, pour les mesures présentées ci-dessus, il est nécessaire de travailler dans des conditions telles que le rendement de la 
diffraction acousto-optique demeure faible, afin de s'assurer de la linearité de la détection par sonde laser, ou d'effectuer les corrections correspondantes quand cela s'avère indispensable, notamment pour 1 'onde acoustique fondamentale et à des niveaux de puissance incidente êlevês.

Les résultats de la figure 4 permettent de déduire le facteur de mérite non linéaire du verre SF-59:

$$
M_{a}=1,1.10^{-12} \mathrm{~s}^{3} / \mathrm{Kg}
$$

valeur très proche de celle trouvée auparavant pour le molybdate de plomb $/ 5 /$.

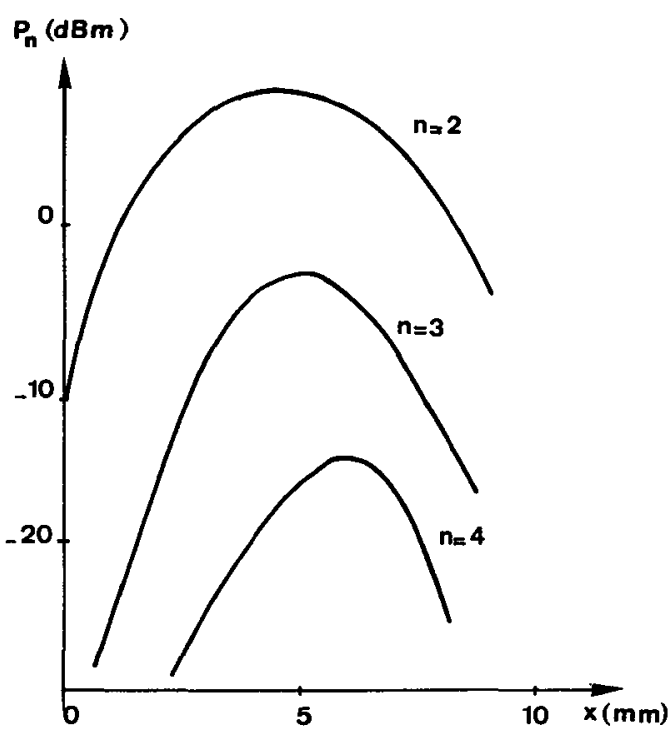

Fig. 4 Puissance des ondes acoustiques harmoniques Pn pour une puissance acoustique d'entrée au niveau du transducteur $\mathrm{Pin}=30 \mathrm{dBm}$ n est le rang de 1 'harmonique

Afin de faciliter les comparaisons, nous donnons dans une table les facteurs de mérite non linéaire $M_{a}$ exprimés en unités $10^{-12} \mathrm{~s}^{3} / \mathrm{kg}$ et les atténuations des ondes acoustiques exprimées en $\mathrm{dB} / \mathrm{cm}$ aux fréquences de travail expérimentales $f$ exprimée en $\mathrm{MHz}$.

TABLE

COMPARAISON DE DIVERS MATERIAUX NON LINEAIRES

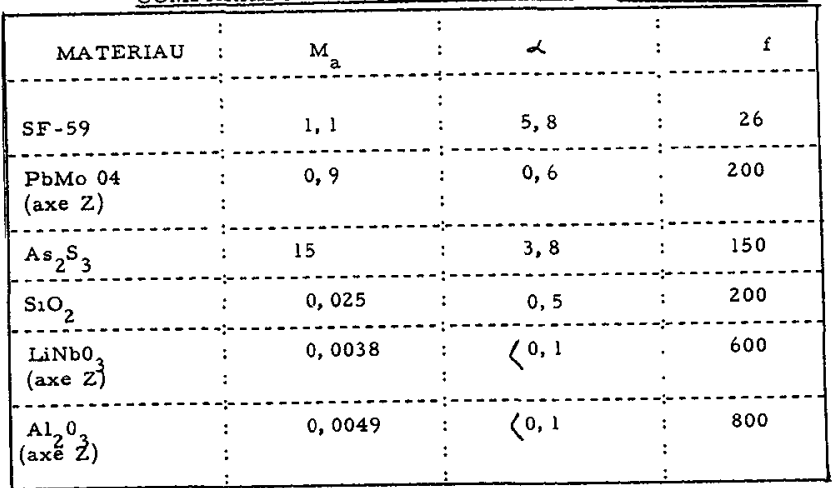

En maintenant la puissance acoustique incidente a. un niveau suffisamment faible pour que l'exès d'atténuation dû aux effets non linéaires demeure non négligeable (OdBm), nous avons relevé les variations de l'atténuation intrinsèque du milieu avec la fréquence. Les résultats sont représentés à la figure 5 et sont en accord moyen avec ceux publiês antérieurement $/ 3 /$, les faibles écarts pouvant s'expliquer par le fait que nous avons corrigé la courbe afin de tenir compte des pertes par diffraction.

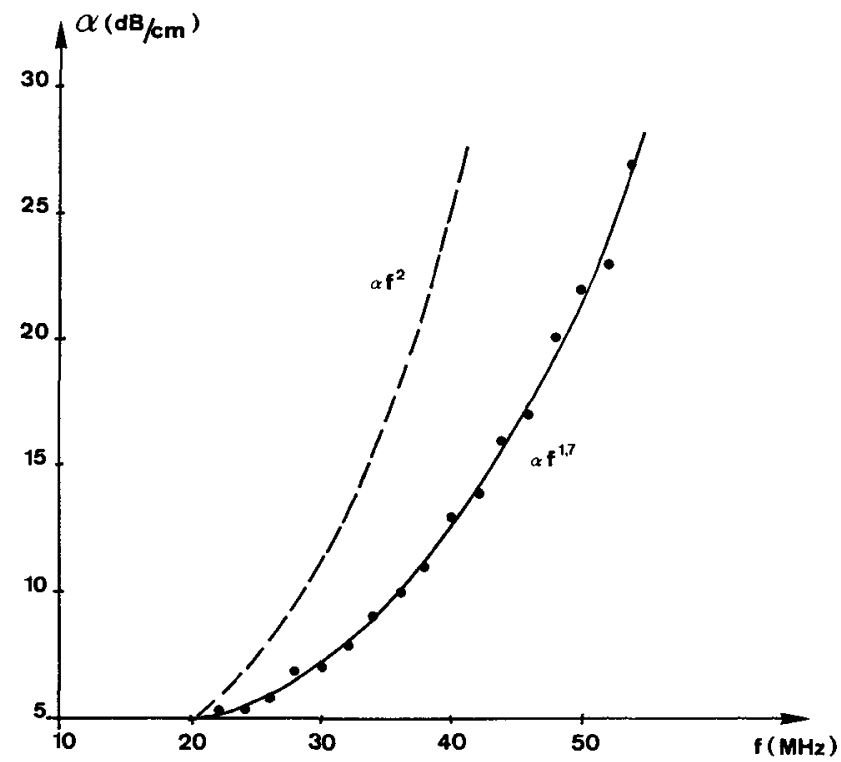

Fig. 5 Atténuation intrinsẽque du verre SF-59 -.. 10 i quadratique

$$
10 \text { i réelle }
$$

3. CONCLUSION. - L'utilisation du verre SF-59 en acoustique non linéaire peut être envisagée, notamment dans le domaine du traitement du signal $/ 7 /$. Cependant, les fréquences opératoires doivent être choisies suffisamment basses pour que l'atténuation des ondes acoustiques ne devienne pas prohibitive. Il est ainsi possible de réaliser des composants de traitement du signal de performances légèrement inférieures à ceux en molybdate de plomb monocristallin ou en verres chalcogénides comme le trisulfure d'arsenic $/ 8 /$, ce qui diminue très sensiblement le prix de revient. 


\section{References}

/1/ N. Uchida et N. Nuzeki, Proc I.E.E.E. (1973)61, 1073

/2/ E.I. Gordon, Proc. I.E.E.E., (1966), 54,1391

/3/ H.Eschler et F. Weidinger, J. AppT. Phys. (1975) 46,65

14/ E.I. Gordon, I.E.E.E. Quant Electron, QE-2(1966) 104

/5/ J.M. Rouvaen, E. Bridoux, M. Moriamez et R. Torquet, App]. Phys. Lett., (1974) 25, 97

/6/ J.M. Roúvaen et E. Bridoux, Rapport final du Contrăt B.R.M.E. $n^{\circ} 751076$ "Systèmes Acoustooptiques de contrôle des Microcircuits Acoustiques" (1975)

17/ E. Bridoux, JM. Rouvaen, C. Bruneel et R. Torguet, J. Appl. Phys. (1975) 46, 2384

18/ J.M. Rouvaen et E. Bridoux Rapport Final A.T.P. Dispositifs Electroniques "Interaction paramétriques entre ondes acoustiques" n० 2343 (1978). 